\title{
SALUD Y TRABAJO DOCENTE - UNA INVESTIGACIÓN CUALITATIVA
}

\author{
Ana Maria Olivera Recarte \\ Consejo de Educación Secundaria, Administración Nacional de Educación Pública, Uruguay \\ aolivera@ces.edu.uy; olivera.recarte@gmail.com
}

\begin{abstract}
Resumen. Investigación de carácter cualitativo que analizó las percepciones de docentes de Ciclo Básico en liceos públicos de Uruguay acerca de las condiciones y medio ambiente de trabajo y su vínculo con la salud. Se identificaron factores de riesgo y satisfactores del trabajo desde el enfoque teórico de Campo, Habitus y Capital de Bourdieu junto al marco analítico de las Condiciones y Ambiente de Trabajo (CyMAT) de OIT. En una primera etapa se entrevistó a directores de cuatro centros seleccionados y se observó el accionar del profesorado en las instituciones visitadas. En segundo lugar, se realizaron entrevistas en profundidad a docentes efectivos con más de cuatro años de ejercicio y menos de veinte años de antigüedad de diferentes sectores del conocimiento Ciencias Sociales, Arte y Expresión y Ciencias Experimentales. La principal técnica utilizada fue la entrevista en profundidad cuyo producto narrativo fue sometido al análisis de contenido convencional a partir del cual se crearon seis dimensiones analíticas: $a$ : Dificultades que encuentra en el liceo para cumplir con la planificación educativa; $b$ : El problema de los espacios; $c$ : El problema del tiempo, $d$ : Satisfacción con el trabajo, e: Carga laboral síquica y física y f: La Evaluación como problema.
\end{abstract}

Palabras clave: Condiciones y Medio Ambiente de Trabajo; Salud y Trabajo docente.

\section{HEALTH AND TEACHING WORK - QUALITATIVE RESEARCH}

\begin{abstract}
Qualitative research where the perceptions of teachers of Basic Cycle in public schools in Uruguay about working conditions and environment and their link with health were analyzed. Risk factors and job satisfaction were identified from the theoretical approach of Field, Habitus and Capital of Bourdieu in dialogue with the analytical framework of Conditions and Work Environment (CyMAT). In a first stage, directors of four selected centers were interviewed and the actions of teachers in the institutions visited were observed. Secondly, in-depth interviews were conducted with effective teachers with more than four years of exercise and less than twenty years old from different sectors of knowledge Social Sciences, Art and Expression and Experimental Sciences. The main technique used was the in-depth interview whose narrative product was subjected to the analysis of conventional content from which six analytical dimensions were created: a: Difficulties found in the high school to comply with educational planning; b: The problem of spaces; c: The problem of time, $\mathrm{d}$ : Satisfaction with work, e: Psychic and physical workload and f: Evaluation as a problem.
\end{abstract}

Keywords: Conditions and Working Environment; Health and Teaching Work.

\section{INTRODUCCIÓN}

La Educación en Uruguay se rige por la ley 18437 que fue promulgada el 12 de diciembre de 2008 por el Poder Ejecutivo donde se establecen los fines, objetivos y orientación de la política educativa en todos sus niveles, incluida la educación no formal y habilitación y supervisión de los institutos privados de enseñanza. En marzo de 2016 la ANEP desarrolló el Sistema de Protección de Trayectorias Educativas (SPTE) que incluye un Protocolo de actuación para los distintos actores de cada Centro y así lograr la continuidad de los Ciclos Educativos. Este programa ha significado el seguimiento y monitoreo de cada cohorte de Enseñanza Media Básica (EMB) a fin de evitar la 
desvinculación primero cognitiva y luego institucional del estudiante. Para ello el Consejo Directivo Central (CODICEN), órgano de dirección de la enseñanza secundaria pública en el país, ha dispuesto la creación de las Unidades Coordinadoras de Integración Educativa (UCDIE), se trata de equipos departamentales que conjuntamente con las estructuras organizativas ya existentes en la Administración Nacional de Educación Pública (ANEP, terciaria) trabajan coordinadamente en establecer aquellos espacios y acciones dirigidos al desarrollo exitoso de las políticas educativas.

Con su creación se pretende fortalecer los procesos de desconcentración de las políticas educativas, contribuir a la concreción de un modelo cohesionado de integración educativa en clave territorial, potenciar la coordinación de la educación formal y no formal y la participación de todos los actores en cada territorio. La institución educativa estructura la construcción de lo común mediante la escolarización que implica pasar de un aprendizaje incidental a un aprendizaje intencional. A medida que avanza el alumno en los niveles de enseñanza especialmente en la secundaria las exigencias de abstracción, descontextualización, controlar y aprender a aprender son mayores.

Es en este tramo del desarrollo de creciente autonomía de los/as adolescentes donde se necesita contención emocional y cuando no se produce la satisfacción de las expectativas termina generando la desvinculación institucional. En el contexto aludido ¿Cómo aproximarse a la relación entre la salud y el trabajo docente?

En dicha pregunta se encuadra el tema de la presente investigación. El trabajo en sí mismo constituye un determinante social de la salud y la calidad de vida.

Existe importante evidencia empírica respecto a que los indicadores de salud, bienestar y enfermedad tienen una vigorosa relación con los diferentes procesos de trabajo. Modelos de causalidad en salud laboral asumen que la preservación del estado de salud y los daños a la misma están asociados con las condiciones en que las personas realizan su trabajo.

Es un espacio social de múltiples interacciones ya sean de orden sistémico, individual, colectivo o biológico. Los indicadores referidos a factores de riesgo presentes en los puestos de trabajo son entre otros las condiciones de empleo y la organización del proceso de trabajo. Además, suelen existir recursos y acciones preventivas 
desarrolladas por empresas y trabajadores asociadas a diferentes estrategias para el afrontamiento de los eventuales daños a la salud derivados del trabajo.

En el campo del trabajo docente interesa estudiar y comprender la constitución y el posicionamiento del sujeto, el proceso de trabajo y el amplio entorno donde el mismo es ejecutado. En este punto es particularmente aplicable el enfoque teórico de Pierre Bourdieu dado que en el campo de la educación a una multiplicidad de actores se agrega una multiplicidad de campos edificando un constructo de relaciones sociales particularmente complejo donde los actores ponen en juego su capital, entendido este como total y constituido por los capitales económico, cultural, simbólico y social. Bourdieu (1997).

El marco analítico que propone Bourdieu no da cuenta de un mundo idealizado sino al contrario, muestra a los actores disputando con su habitus los espacios de poder donde su capital (simbólico, social, cultural o económico) les permita ubicarse. Seguramente el "docente tipo" ocupe un lugar relativamente importante en la escala de lo simbólico y lo cultural y una posición débil en términos de capital social y económico y no cuesta pensar que la variable salud ha de ser, ora estrategia defensiva, ora estrategia de sobrevivencia en un campo hostil donde seguramente se encuentra en la posición más débil como trabajador asalariado sujeto a jerarquía.

El docente en tanto actor social construye percepciones sociales acerca de su mundo laboral donde lleva adelante su quehacer educativo y de las prácticas que en él ocurren. A partir de aquí surgen interrogantes: ¿Qué aspectos de la organización del trabajo pueden ser advertidos por los actores involucrados como principal o principales causas de daño a la salud? ¿Hasta qué punto el malestar docente forma parte de la explicación o del discurso de los actores involucrados?

En este caso se propone asociar al concepto de malestar docente la definición de Salud: Según la Organización Mundial de la Salud (OMS), en una definición de amplia divulgación en estos tiempos, el concepto de salud se entiende como un "estado completo de bienestar físico, mental y social" no consistiendo solo en la ausencia de enfermedad (Alcántara, 2008).

Por otro lado, y complementario al concepto de Salud asociado al proceso de trabajo están las Condiciones y Medio Ambiente de Trabajo (CyMAT) que comprende a todos 
los elementos reales que inciden directa o in/directamente en la salud de las y los trabajadores, constituyendo un conjunto que obra en la realidad concreta de la situación laboral (Neffa, 2015).

\section{OBJETIVOS}

Principal: Analizar las percepciones de los docentes de enseñanza secundaria sobre la relación entre la organización del trabajo docente y daños a la salud. Especificos: Asociados a las preguntas de investigación:

1:¿Cómo se percibe el sorteo de las dificultades que se encuentran en el liceo para cumplir la planificación educativa y encontrar satisfactores en el trabajo?

2: ¿Cómo se percibe la disposición de tiempos institucionales para el aprendizaje de las y los estudiantes?

3: ¿Cómo se observan los espacios físicos del liceo respecto a su adecuación para cumplir la tarea docente?

4: ¿Cómo impactan los tiempos institucionales de aula, coordinación y recreación respecto a su adecuación para el buen desempeño profesional?

\section{MÉTODOS}

Para la recolección de información la técnica principal fué la entrevista en profundidad. La muestra, en la etapa de selección de casos tuvo carácter intencional sin pretensión de representatividad estatística sino que estuvo signada por la búsqueda de relatos sustantivos en relación a la temática de investigación planteada (Flick, 2012). El trabajo de campo se realizó en los meses de octubre-noviembre de 2017 en la jurisdicción Norte 2 del CES que comprenden los departamentos de Rivera, Tacuarembó y Cerro Largo. Se realizaron 12 entrevistas en profundidad a docentes mujeres en funciones de docencia directa, tituladas y efectivas. Dichas entrevistas fueron desgrabadas y procesadas a través del MAXQDA 2018, software aplicado a la investigación cualitativa. Se realizó análisis de contenido convencional y para ello el corpus discursivo de las entrevistas fue objeto de un proceso de etiquetado por segmentos de acuerdo a definiciones analíticas previas.

\subsection{Resultados}


Análisis de las narrativas de los actores. A efectos de operativizar el análisis narrativo se crearon dimensiones analíticas a partir de los indicadores micro de las condiciones y medio ambiente de trabajo:

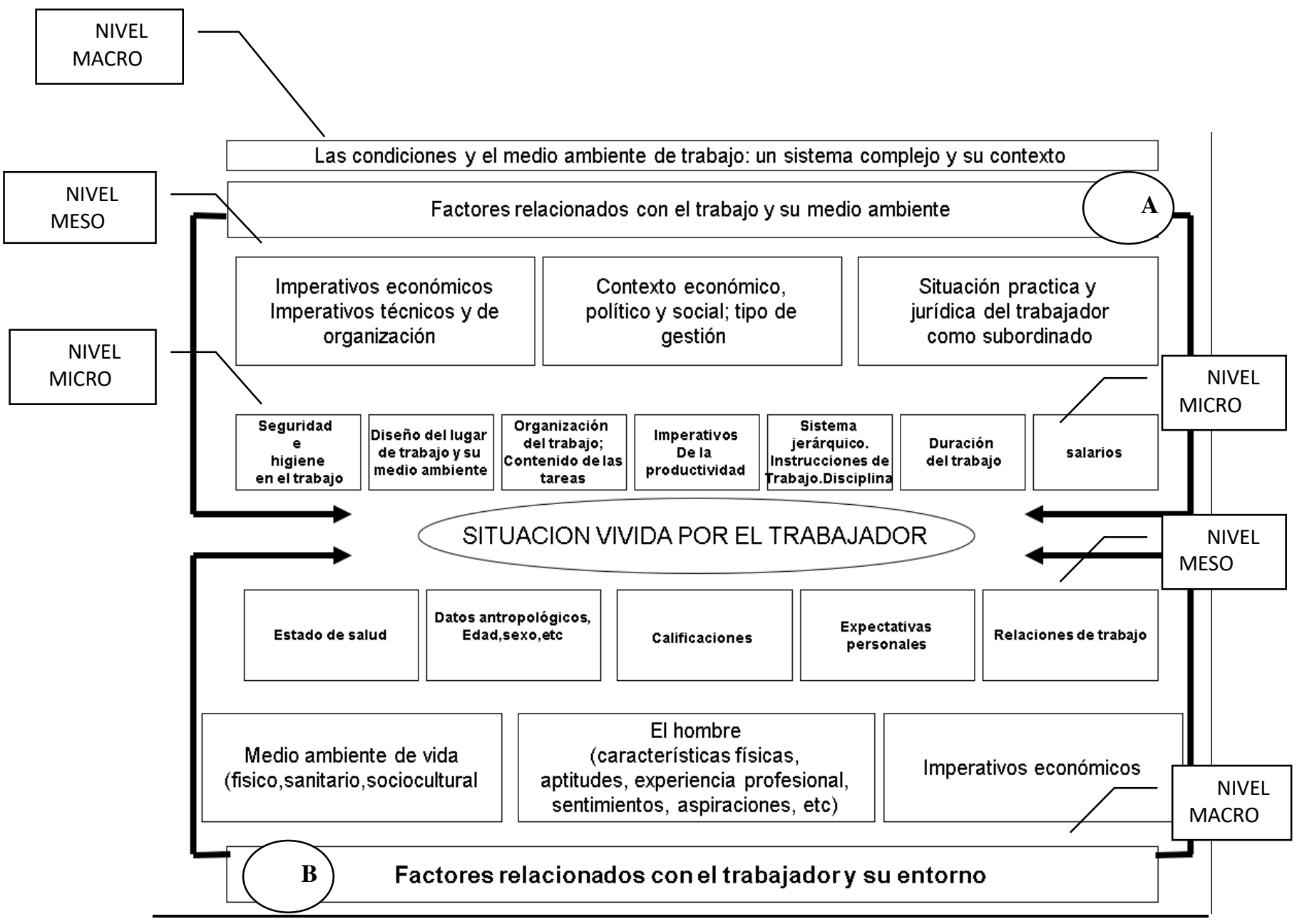

Figura 1. Esquema rector del análisis. Las CyMAT

Fuente: Material didáctico/DSO. Departamento de Salud Ocupacional de Facultad de Medicina. UdelaR

Una vez conceptualizados los indicadores micro de las CyMAT abordar la narrativa de los entrevistados supuso una lectura recursiva y reflexiva de las entrevistas realizadas en 2 etapas sucesivas y diferenciadas.

- Etapa A: Detectar tópicos narrativos a partir de las definiciones operativas de los indicadores micro de CyMAT. 
- Etapa B: Establecer dimensiones analíticas que dieran cuenta de los objetivos de la investigación a partir de vincular definiciones teóricas previas con la evidencia empírica construida.

Cuadro 1. Tópicos narrativos y dimensiones analíticas

\begin{tabular}{|c|c|c|}
\hline $\begin{array}{c}\text { Tópicos narrativos a partir de } \\
\text { las definiciones operativas de } \\
\text { los indicadores micro de CyMAT } \\
\text { (Dimensión A) }\end{array}$ & $\begin{array}{c}\text { Tópicos narrativos a partir de } \\
\text { las definiciones operativas de } \\
\text { los indicadores micro de CyMAT } \\
\text { (Dimensión B) }\end{array}$ & $\begin{array}{c}\text { Dimensiones analíticas entre } \\
\text { categorías teóricas y evidencia } \\
\text { empírica construida }\end{array}$ \\
\hline Aspectos salariales & Relaciones de trabajo & $\begin{array}{r}\text { Dificultades que encuentra en el } \\
\text { liceo para cumplir con la } \\
\text { planificación educativa }\end{array}$ \\
\hline Duración del trabajo/ carga horaria & Expectativas personales & El problema de los Espacios \\
\hline Sistema jerárquico & Sistema de & El problema del Tiempo \\
\hline Imperativos de productividad & Género y edad & Satisfacción con el Trabajo \\
\hline Contenido de las tareas & Aspectos vinculados a la salud & Carga laboral síquica y física \\
\hline
\end{tabular}

\subsection{Hallazgos principales}

El propósito de esta investigación consistió en relevar y analizar sin pretensiones generalizadoras las percepciones de los docentes de enseñanza media de los centros estudiados acerca de las condiciones de trabajo y su impacto en la salud. Se constató una relación intensa entre el proceso de salud enfermedad de los trabajadores de docencia directa y la forma en que dicho proceso está determinado por la condiciones y medio ambiente de trabajo. Las percepciones del universo de vida investigado tienen su expresión empírica en las dimensiones analíticas construidas a los efectos del análisis:

- La planificación educativa. El discurso docente identifica claramente aquellos elementos que dificultan el trabajo docente y existe una verificable búsqueda de estrategias de facilitación por parte de quienes viven su trabajo día a día;

- Los Espacios. Existe una negociación permanente del uso de los espacios del liceo con otros actores institucionales y se verifica una necesidad sentida respecto a la creación de liceos pensados para el currículo del siglo XXI;

- EI Tiempo. El ejercicio de la práctica docente está atravesado por una tensión permanente por el "tiempo que falta". Falta tiempo para: el contacto diario con el estudiante, en la vinculación con el trabajo colaborativo con otros actores, para la 
corrección, planificación y descanso, en el vínculo con el contacto con los padres y la comunidad educativa. El factor tiempo siempre aparece como una carga psíquica significativa del trabajo docente. Signos y síntomas de carácter somático se expresan en narrativas donde la falta de tiempo es una alusión de carácter permanente;

- Satisfacción con el Trabajo. Es el lugar donde cobran un peso significativo los satisfactores de la profesión. Los mismos cubren el amplio espectro que van desde el trabajo de aula a la planificación y en el sentido dinámico de los saberes que circulan en la organización. Dichos satisfactores contemplan además la posición que ocupa la profesión en las representaciones sociales y es motivo del compromiso que se detecta en la percepción de los docentes, orientados a una búsqueda permanente de la mejora de la función docente y de la identidad profesional personal y colectiva. Carga laboral síquica y física. Tal como señalamos respecto "El Tiempo" la carga física y síquica del trabajo son los conectores más robustos de la compleja relación entre el trabajo docente y el continuo salud- enfermedad de los docentes, sea en su expresión individual como colectiva. Allí además se agrega un componente esencial: el corte de género que radicaliza algunas expresiones del desgaste de las trabajadoras: las mujeres docentes tienen a su cargo tareas de cuidado familiar que incrementan el trabajo diario. Los síntomas físicos que asocian las docentes a su tarea cotidiana son, entre otros, cefaleas persistentes, dolores de columna, osteoarticulares, pérdida de visión y un dato de enorme sensibilidad profesional: la pérdida de la voz;

- La Evaluación. La evaluación del docente es un proceso complejo donde se instalan lógicas inherentes a la estructura de jerarquía laboral y de relaciones entre trabajadores, ya sea en un nivel vertical como horizontal. Relaciones humanas, profesionales, de vínculos y de colaboración ingresan en un territorio volátil y complejo para quienes habitan el campo de la educación (en términos de Bourdieu). Es en el momento de la evaluación donde la noción de capital (sobre todo el capital simbólico y el social) permite ubicar a los individuos en la trama de las posiciones sociales. Si bien es una noción con un fuerte contenido abstracto también traduce tensiones que los individuos sienten en sus cuerpos. Los términos de una evaluación tienen consecuencias duras (buenas o malas) sobre la carrera docente.

\section{CONCLUSIONES}

El propósito de esta investigación consistió en relevar y analizar sin pretensiones generalizadoras las percepciones de los docentes de enseñanza media de los centros estudiados acerca de las condiciones de trabajo y su impacto en la salud. Se concluye que los resultados principales de investigación plantean algunas sugerencias o recomendaciones para la mejora de la salud de los docentes y sus condiciones y medio ambiente de trabajo. En 
cuanto al problema de investigación se constató una relación intensa entre el proceso de salud enfermedad de los trabajadores de docencia directa y la forma en que dicho proceso está determinado por la condiciones y medio ambiente de trabajo. Las percepciones del universo de vida investigado tienen su expresión empírica en las dimensiones analíticas construidas a los efectos del análisis:

En relación a la planificación educativa el discurso docente identifica claramente aquellos elementos que dificultan el trabajo docente y existe una verificable búsqueda de estrategias de facilitación por parte de quienes viven su trabajo día a día. Sobre los espacios debe señalarse que existe una negociación permanente del uso de los mismos en el liceo. Ello ocurre con otros actores institucionales y se verifica una necesidad sentida respecto a la creación de liceos pensados para el currículo del siglo XXI.

El ejercicio de la práctica docente está atravesado por una tensión permanente por el "tiempo que falta". Falta tiempo para: el contacto diario con el estudiante, en la vinculación con el trabajo colaborativo con otros actores, para la corrección, planificación y descanso, en el vínculo con el contacto con los padres y la comunidad educativa.

El factor tiempo siempre aparece como una carga psíquica significativa del trabajo docente. Signos y síntomas de carácter somático se expresan en narrativas donde la falta de tiempo es una alusión de carácter permanente. Un lugar sustantivo y destacable son los satisfactores de la profesión. Los mismos cubren el amplio espectro que van desde el trabajo de aula a la planificación y en el sentido dinámico de los saberes que circulan en la organización. Dichos satisfactores contemplan además la posición que ocupa la profesión en las representaciones sociales y es motivo del compromiso que se detecta en la percepción de los docentes, orientados a una búsqueda permanente de la mejora de la función docente y de la identidad profesional personal y colectiva.

Tal como señalamos respecto "al tiempo" la carga física y síquica del trabajo son los conectores más robustos de la compleja relación entre el trabajo docente y el continuo saludenfermedad de los docentes, sea en su expresión individual como colectiva. Allí además se agrega un componente esencial: el corte de género que radicaliza algunas expresiones del desgaste de las trabajadoras: las mujeres docentes tienen a su cargo tareas de cuidado familiar que incrementan el trabajo diario. Los síntomas físicos que asocian las docentes a su tarea cotidiana son, entre otros, cefaleas persistentes, dolores de columna, osteoarticulares, pérdida de visión y un dato de enorme sensibilidad profesional: la pérdida de la voz. 
La evaluación del docente es un proceso complejo donde se instalan lógicas inherentes a la estructura de jerarquía laboral y de relaciones entre trabajadores, ya sea en un nivel vertical como horizontal. Relaciones humanas, profesionales, de vínculos y de colaboración ingresan en un territorio volátil y complejo para quienes habitan el campo de la educación (en términos de Bourdieu). Es en el momento de la evaluación donde la noción de capital (sobre todo el capital simbólico y el social) permite ubicar a los individuos en la trama de las posiciones sociales. Si bien es una noción con un fuerte contenido abstracto también traduce tensiones que los individuos sienten en sus cuerpos. Los términos de una evaluación tienen consecuencias duras (buenas o malas) sobre la carrera docente. La relación entre el trabajo docente y su vínculo con la salud del trabajador aparece como un campo necesario de recolección de evidencia empírica y conformación de masa crítica a efectos de intervenir en las claves explicativas del denominado "malestar docente"1.

En ese sentido, las percepciones de los trabajadores en torno a la salud y la enfermedad aparecen como un espacio adecuado para generar reflexión teórica orientada a comprender los procesos sociales que habiliten la mejora de las condiciones del trabajo docente.

En razón de dar cumplimiento a la Ley 18.508 que refiere a la Negociación Colectiva en el marco de las relaciones laborales y en virtud de que debe cumplirse con el Decreto 291/007 que reglamenta el Convenio Internacional de Trabajo Na155 ratificado por la ley 15.965 del 28 /6/1988 el Consejo de Educación Secundaria , con fecha 30 de julio de 2013 conforma una Comisión de Salud Laboral de carácter bipartito con delegados del CES, FENAPES y Asociación de Trabajadores de Enseñanza Secundaria ( ATES) que exhorta a promover la salud de los trabajadores con comisiones por centro educativo, con titular y suplente. En sus cometidos está la planificación de la prevención combatiendo los riesgos laborales en su origen, promover y mantener la cooperación en salud, seguridad y ambiente laboral.

La principal recomendación de esta investigación es la aplicación sistemática de dicho marco normativo. Debe señalarse que ello incluye revisar en forma consensuada entre los actores del quehacer educativo la relación adecuada entre la salud y el trabajo docente como una condición inexcusable para la generación de un clima organizacional de los liceos públicos que propenda a la mejora continua de la oferta educativa. Sabedores de que el ejercicio consciente y responsable de los cargos de Gestión pública por sí solos no modifican

\footnotetext{
${ }^{1}$ Refiere a la obra de José Manuel Esteve (1997) cuyo título se ha transformado en una metáfora recurrente para aludir a la relación entre la salud y el trabajo docente
} 
estructuras tradicionales de ver y verse como trabajadores docentes, es necesario la facilitación de la política educativa para el encuentro e implementación de los cambios necesarios.

\section{REFERENCIAS}

Alcántara, M. G. (2008). La definición de salud de la Organización Mundial de la Salud y la interdisciplinariedad. Sapiens. Revista Universitaria de Investigación, 9(1)93-107.

ANEP (2007). Censo Nacional Docente ANEP - 2007. Disponible en : http://observatorio.anep.edu.uy/images/documentos/relevamientos/Censo Nacional Docente 2007.pdf

Bourdieu, P. (1997). Razones prácticas. Sobre la teoría de la acción. Barcelona: Anagrama. ISBN: 84-339-05430 .

CES. (2018). Monitor Educativo. Disponible en: http://servicios.ces.edu.uy/monitorces/servlet/portada.

Flick, U. (2012). Introducción a la investigación Cualitativa. Morata: A Coruña, España.

Neffa, J. C. (2015). Introducción al concepto de Condiciones y Medio Ambiente de Trabajo (CyMAT). Editorial: Universidad de Buenos Aires. Facultad de Ciencias Económicas en Revista: Voces del Fénix/ISSN: 1853-8819.

Robalino, M., \& Körner, A. (2005). Condiciones de trabajo y salud docente estudios de casos en Argentina, Chile, Ecuador, Mexico, Peru y Uruguay. Coordinación: Magaly Robalino Campos y Antón Körner/ Publicado por la Oficina Regional de Educación de la UNESCO para América Latina y el Caribe, OREALC / UNESCO Santiago de Chile, Chile.

Tedesco, J. C. (2008). ¿Son posibles las políticas de subjetividad? en Nuevos temas en la agenda de política educativa. Siglo XXI: Buenos Aires.

Tomasina. (2005). Estudio de caso en Uruguay Universidad de la República, Uruguay Facultad de Medicina Departamento de Salud Ocupacional en: condiciones de trabajo y salud docente estudios de casos en Argentina, Chile, Ecuador, Mexico, Peru y Uruguay (pp. 197-211). Coordinación: Magaly Robalino Campos y Antón Körner. 